\title{
Screening of Polymer Additives in Drinking Water Stored in PET Bottles by UHPLC-ESI-IT-TOF MS
}

\author{
Mustafa Abdullah Yilmaz ${ }^{1,2}$ and Hamdi Temel ${ }^{1,2}$ \\ 1. Dicle University Science and Technology Research and Application Center (DÜBTAM), Dicle University, Diyarbakır 21280, \\ Turkey \\ 2. Department of Pharmaceutical Chemistry, Faculty of Pharmacy, Dicle University, Diyarbakır 21280, Turkey
}

\begin{abstract}
In this study, Ultra High Performance Electrospray Ionization Ion Trap Time of Flight Mass Spectrometry (UHPLC-ESI-IT-TOF MS) method is used to screen the polymer additives in drinking water that is stored in Polyethylene Terephthalate (PET) bottles. After directly analyzing the commercially available water samples, 3 polymer additives (chimassorb 81, irgafos 168 and oleamide) were detected. However, after exposure to sunshine for 8 days, two additional polymer additives (antioxidant 2246 and Butylated Hydroxytoluene (BHT)) were detected besides the former three additives.
\end{abstract}

Key words: UHPLC-ESI-IT-TOF MS, polymer additives, PET bottle, drinking water, health hazards.

\section{Introduction}

Plastics are inevitable and ubiquitous materials in todays' world. Over the last 20-30 years, the usage of polymeric materials have been significantly increased in most of the applications of daily life and industry. Different types of polymers are used in thousands of applications, from construction to electronic devices to food and beverage packaging [1]. Since polymer types and uses has expanded, formulations become increasingly complex. That is to say, today's commercially available plastics are quite sophisticated materials. That's because new applications almost always require additives to increase performance properties such as toughness, flexibility, conductivity, antistatic qualities and lifetime. Polymer manufacturers add antioxidants, Ultraviolet (UV) light absorbers, stabilisers and other materials to enhance polymer utility or processing. A variety of polymers are used as food-contact plastic materials. Polyethylene Terephthalate (PET) is a semi-crystalline polymer which belongs to the family of polyesters. It

Corresponding author: Mustafa Abdullah Yilmaz, associate Ph.D., main research field: mass spectrometry based metabolomics. is the most suitable packaging material for drinking water. PET bottles have been marketed for the last four decades and they have gradually replaced Polyvinylchloride (PVC) and glass bottles on the markets. A recent European Regulation No. 10/2011 [2] covers food-contact plastic materials. It states that food-contact materials should not transfer its ingredients to food in quantities that could incur a human health risk, cause an unacceptable change in the composition of the food or bring about deterioration in the food organoleptic characteristics $[3,4]$.

There are several polymer additives such as antioxidants, UV absorbing agents and stabilizators to enhance the performance of PET bottles. Below are some of these polymer additives used $[3,5,6]$ :

- Antioxidant agents: Intioxidant 2246, BHT, Irganox MD 1024, Irganox 1081, Irganox 1035, irganox 3114, irganox 1010, irganox 1330, irganox 1076, Ultranox 626 and Weston 399;

- UV absorbing agents: chimassorb 81, Tinuvin 234, Tinuvin 326, Tinuvin 327 and Tinuvin 328;

- Stabilizers: irgafos 126, irgafos 38 and irgafos 168 , erucamide and oleamide. 
It is important to quantify and qualify these additives for quality control and troubleshooting. Identification of polymer additives is desired if competitor products are investigated or degradation processes are studied. Both quantification and identification are difficult tasks because there are a wide variety of different additives, usually mixtures of additives are used, and the amount added is often low and can be further decreased due to degradation. That is the case when analysing the polymer additives in the polymer itself [3]. The identification of polymer additives inside the water that has a polymer package is even more difficult. Mass spectrometric detection can solve this problem because it is highly sensitive and structural information can be derived from the mass spectra. In previous studies, several chemicals and additives such as phthalates, alkylphenols, adipates and BisPhenol A (BPA) have been investigated in baverage, water and food packaging materials by using different techniques $[7,8]$.

In this study, a number of polymer additives in drinking water stored in PET bottles were screened by a unique UHPLC-ESI-IT-TOF MS instrument. As a result of this study, five polymer additives which are used to enhance the quality of the PET bottles were detected. Three of them (chimassorb 81, irgafos 168 and oleamide) were detected after directly analyzing the water. However, after exposing the drinking water inside the PET bottle to sunshine, two additional polymer additives (antioxidant 2246 and BHT) were detected.

\section{Material and Methods}

Acetonitrile (chromatographic grade) and ammonium acetate (analysis grade) were purchased from Sigma Aldrich. Ultrapure water was freshly prepared with the sartorious water purification system. Commercially, available drinking water samples stored in PET bottles were purchased from a local store. Finisterre C18, $6 \mathrm{~mL}, 500 \mathrm{mg}$ Solid Phase Extraction (SPE) cartridge was used and purchased from Teknokroma.

A simple solid phase extraction procedure was applied to concentrate polymer additives in drinking water samples. SPE sorbents were first conditioned by washing $10 \mathrm{~mL}$ acetonitrile and followed by $10 \mathrm{~mL}$ of ultrapure water. Then drinking water samples were applied $(100 \mathrm{~mL})$ through the SPE cartridges by employing a moderate vacuum at about $10 \mathrm{~mL} / \mathrm{min}$. After that, SPE cartridges were air dried for $10 \mathrm{~min}$ under vacuum to remove as much residual water as possible. The compounds were eluted with $1 \mathrm{~mL}$ acetonitrile twice. The acetonitrile fraction was collected and evaporated under a stream of nitrogen at $40{ }^{\circ} \mathrm{C}$, and the dry residue was reconstituted with 1 $\mathrm{mL}$ acetonitrile-water $(1: 1, \mathrm{v} / \mathrm{v})$. Finally, $5 \mu \mathrm{L}$ of the last solution was directly injected into the UHPLC-ESI-IT-TOF MS system for analysis.

Polymer additives were identified by a Shimadzu UHPLC-ESI-IT-TOF MS hybrid mass spectrometer. The liquid chromatography system (Shimadzu) was equipped with a solvent delivery pump (LC-20AD), an autosampler (SIL-20AC), a DGU-20A3 degasser, a photodiode array detector (PDA and SPD-M20A), a communication base module (CBM-20A) and a column oven (CTO-20AC). The separation was performed without column using a gradient elution consisting of mobile phase A $(0.1 \%$ formic acid, 5 $\mathrm{mM}$ ammonium acetate and water) and mobile phase B $(0.1 \%$ formic acid and acetonitrile). The gradient was: $0-10 \mathrm{~min}$, a linear gradient from $50 \%$ B to $100 \%$ B; 10-15 min, $100 \% \mathrm{~B}$ and 15-20 min, a linear gradient back to $500 \% \mathrm{~B}$. The whole analysis took 20 min. The sample chamber in the autosampler was maintained at $4{ }^{\circ} \mathrm{C}$. The injection volume was $5 \mu \mathrm{L}$, the flow rate was $0.3 \mathrm{~mL} / \mathrm{min}$, and the Photodiode Array (PDA) detection was performed from $200 \mathrm{~nm}$ to $400 \mathrm{~nm}$. The total effluent from the ESI (Electrospray Ionization) source was transferred directly to the hybrid IT/TOF mass spectrometer without splitting. The mass spectrometer was equipped with an electrospray ionization source and operated in both 
positive and negative ionization mode. The mass range was set at 150-800 Da. Nitrogen was used as the nebulizing gas at a flow rate of $1.5 \mathrm{~L} / \mathrm{min}$. The interface and detector voltages were set at $4.5 \mathrm{kV}$ and $1.61 \mathrm{kV}$, respectively. The Curved Desolvation Line (CDL) and heat block temperatures were both maintained at $200{ }^{\circ} \mathrm{C}$. The MS1 spectra were produced and the ion accumulation time was set at 30 $\mathrm{ms}$. The relative energy was set at $50 \%$.

Data acquisition and processing were carried out using the LC/MS solution version 3.41 software supplied with the instrument. Any mass numbers corresponding to particular elemental compositions were also calculated by the formula predictor, and would generate more than one formula proposed by the software. Therefore, accuracy error threshold of $\pm 10 \mathrm{ppm}$ was set as a limit to the calculation of possible elemental compositions. The other conditions for calculating elemental compositions were taken into consideration: the upper limits on the number of $\mathrm{C}, \mathrm{H}$, $\mathrm{O}, \mathrm{N}, \mathrm{P}$ atoms, $\mathrm{C} / \mathrm{H}$ ratios, nitrogen rule and range of double-bond equivalent.

\section{Results and Discussion}

Several previous studies reported the presence of plasticier, polymer additive and polymer related compound residues in bottled water, food or bevarages with plastic packaging. Although trace levels of oligomers ranging from $4.9 \mathrm{mg} / \mathrm{kg}$ to $8.7 \mathrm{mg} / \mathrm{kg}$ in PET bottle-grade is reported, migration studies of oligomers from PET into water haven't been published recently [9]. In this study, also some carbonyl compound (acetaldehyde and formaldehyde) levels in PET bottles from Japan, Europe and North America have been investigated [9]. Despite the fact that phthalates are not supposed to be used in PET bottles, they have been found in PET material and in PET-bottled water. Several studies reported that phthalates may come from bottling lines, cap-sealing resins or water treatment facilities [10-13]. Also, background pollution in the laboratory analyzing phthalates may also be a source [14]. A previous study compared the results of analyses of bottled water before and after storage, and concluded that insufficient storage conditions (10 weeks storage outdoors at temperatures up to $30^{\circ} \mathrm{C}$ ) increases the concentrations of Dibutyl Phthalate (DBP), Benzyl Butyl Phthalate (BBP) and Bis(2-ethylhexyl) Phthalate (DEHP) in PET bottled water [15]. Antioxidants are added to the polymer in small amounts to impede or minimize the oxidation of plastic material. However, PET bottles intended for water are usually manufactured without antioxidants [16]. BHT is a phenolic antioxidant used as a thermostabilizer for Polyethylene (PE), Polypropilene (PP), polyesters and PVC [17]. In a previous study, BHT amounts ranging from $21 \mathrm{mg} / \mathrm{L}$ to $38 \mathrm{mg} / \mathrm{L}$ were found in PET-bottled water [18]. In a further study, only BHT was found in glass-bottled water $(2.5 \mathrm{mg} / \mathrm{L})$ which means the origin of this compound is the PE bottle caps [19]. That is a logical outcome since BHT may be used in the manufacture of PE.

As mentioned before, in this study, several polymer additives in drinking water samples that are commercially available were screened by UHPLC-ESI-IT-TOF MS instrument. Chimassorb 81, irgafos 168 and oleamide were detected without exposing to sunshine. After exposure to sunshine additional two polymer additives antioxidant 2246 and BHT were also detected. It should be kept in mind that, the fact that these polymer additives constantly entering human body may cause serious health problems [2, 4]. For example, it was determined that being a UV absorber chimassorb 81 increases the sensitivity of skin towards allergic deseases and could cause damage to the lungs and kidneys [20]. Nevertheless, oleamide used as a stabilizer in contact with the skin can cause sensitization, which can be irritating to eyes, respiratory system and skin. Repeated exposure of oleamide may cause skin dryness or cracking Additionally, it is toxic to aquatic organisms in the aquatic environment and may cause long-term adverse effects [21]. Moreover, antioxidant 
Table 1 Molecular formulas, molecular structures and exact masses (for $\mathrm{M}, \mathbf{M}+\mathrm{H}^{+}$and $\mathrm{M}-\mathrm{H}^{+}$) of studied polymer additives.

\begin{tabular}{|c|c|c|c|}
\hline Additive & Formula & Structure & Exact masses: $\mathrm{M}, \mathrm{M}+\mathrm{H}^{+}$and $\mathrm{M}-\mathrm{H}^{+}$ \\
\hline Chimassorb 81 & $\mathrm{C}_{21} \mathrm{H}_{26} \mathrm{O}_{3}$ & & $\begin{array}{l}326,1882 \\
327,1955 \\
325,1809\end{array}$ \\
\hline Irgafos 168 oxide & $\mathrm{C}_{42} \mathrm{H}_{63} \mathrm{O}_{4} \mathrm{P}$ & & $\begin{array}{l}662,4464 \\
663,4537 \\
661,4391\end{array}$ \\
\hline Oleamide & $\mathrm{C}_{18} \mathrm{H}_{35} \mathrm{NO}$ & & $\begin{array}{l}281,2719 \\
282,2791 \\
280,2646\end{array}$ \\
\hline Antioxidant 2246 & $\mathrm{C}_{23} \mathrm{H}_{32} \mathrm{O}_{2}$ & & $\begin{array}{l}340,2402 \\
341,2475 \\
339,2330\end{array}$ \\
\hline BHT & $\mathrm{C}_{15} \mathrm{H}_{24} \mathrm{O}$ & & $\begin{array}{l}220,1827 \\
221,1900 \\
219,1754\end{array}$ \\
\hline
\end{tabular}

2246 is harmful if swallowed, irritating to eyes may cause sensitization by skin contact and with all of these, there is a risk of irreversible effects [22]. In addition to that, BHT is hazardous in case of inhalation (lung irritant), eye contact (irritant) and skin contact (irritant), and there is a slight threat if swallowed [2]. Besides, BHT is mutagenic for mammalian somatic cells, bacteria and yeasts. This material could be toxic to the central nervous system, blood and liver. Repeated or prolonged exposure to this substance may produce damage in target organs [23].

Due to its inherent characteristics of accurate mass measurements, UHPLC-ESI-IT-TOF MS instrument was used. Fig. 1 shows the accurate MS1 spectra, exact masses and some important data for the characterization of the polymer additives (chimassorb 81 , irgafos 168 oxide, oleamide, antioxidant 2246 and BHT) by the help of Formula Predictor Software (only Fig. 1 is given in the paper, others were given as supporting information file). Additionally, Table 1 shows the molecular formulas, molecular structures and exact masses (for $\mathrm{M}, \mathrm{M}+\mathrm{H}^{+}$and $\mathrm{M}-\mathrm{H}^{+}$) of five polymer additives that were screened in the study. For chimassorb 81 and BHT, ESI source is operated in negative mode because of the phenolic $\mathrm{OH}$ groups. Nonetheless, for irgafos 168 oxide, oleamide and antioxidant 2246, negative mode was chosen. 

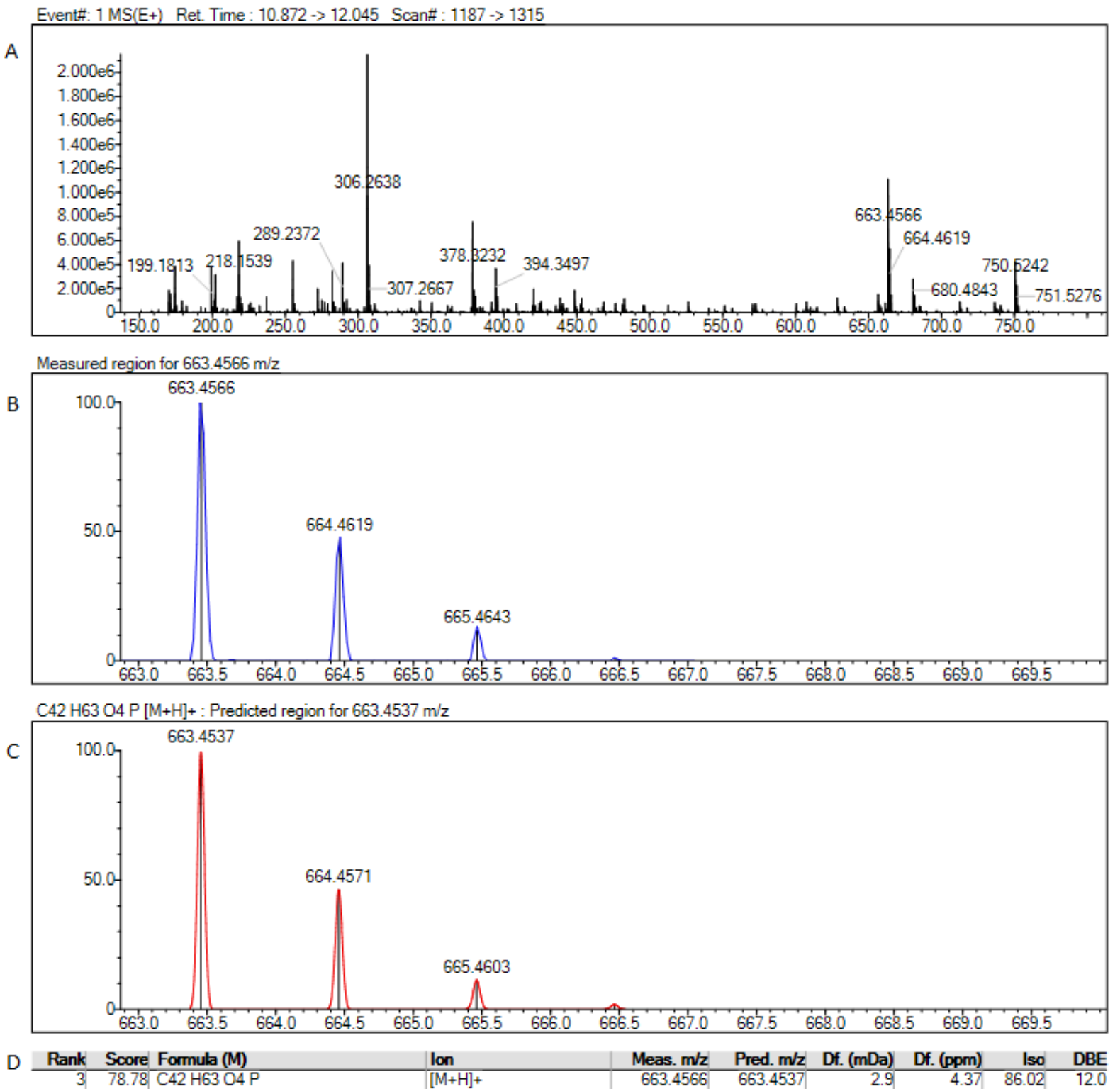

Fig. 1 Single MS spectrum and specific exact mass of irgafos 168 oxide (A is accurate MS1 spectrum of irgafos 168 oxide, B and $\mathrm{C}$ are the spectra that show the measured and predicted exact mass of the compound irgafos 168 oxide respectively by LC-ESI-IT-TOF MS, D is the information about predicted elemental composition, measured accurate mass, theoretical mass, Double Bond Equivalent (DBE) and mass errors of the compound irgafos 168 oxide).

\section{Conclusion}

To conclude, drinking water stored in PET bottles are all around people and they use them frequently in their daily lifes. It was found that five polymer additives transfered from PET to water by a simple method with a unique instrument that is UHPLC-ESI-IT-TOF MS. Three of them (chimassorb 81, irgafos 168 and oleamide) were detected after directly analysing the water samples. However, after exposure to sunshine for 8 days antioxidant 2246 and BHT were also detected. Consequently, it is more healthy to choose glass bottled drinking waters instead of PET bottled ones. If it is inevitable, PET bottled waters shouldn't be exposed to sunshine before use.

\section{Acknowledgements}

The authors are thankful to Dicle University 
Science and Tech-nology Research and Application Center (DUBTAM), Ant teknik and Shimadzu Corporation for its support in this study.

\section{References}

[1] Himmelsbach, M., Buchberger, W., and Reingruber, E. 2009. "Determination of Polymer Additives by Liquid Chromatography Coupled with Mass Spectrometry, A Comparison of Atmospheric Pressure Photoionization (APPI), Atmospheric Pressure Chemical Ionization (APCI), and Electrospray Ionization (ESI), Polym." Degrad. Stab. 94: 1213-1219.

[2] EU. 2011. "Commission Regulation (EU) No 10/2011 of 14 January 2011 on Plastic Materials and Articles İntended to Come in Contact with Food." Official Journal of the European Commission L12: 1-89.

[3] Bach, C., Dauchy, X., Chagnon, M. C., and Etienne, S. 2012. "Chemical Compounds and Toxicological Assessments of Drinking Water Stored in Polyethylene Terephthalate (PET) Bottles: A Source of Controversy Reviewed." Water Research 46: 571-583.

[4] Lithner, D., Larsson, Å., and Dave, G. 2011. "Environmental and Health Hazard Ranking and Assessment of Plastic Polymers Based on Chemical Composition." Sci. Total Environ. 409: 3309-3324.

[5] Pospíšil, J., and Weideli, H. J. 1996. "Environmental İmpacts Associated with the Application of Radical-Scavenging Stabilizers in Polymers." Polym. Degrad. Stab. 52: 109-117.

[6] Block, C., Wynants, L., Kelchtermans, M., De Boer, R., and Compernolle, F. 2006. "Identification of Polymer Additives by Liquid Chromatography Mass Spectrometry.” Polym. Degrad. Stab. 91: 3163-3173.

[7] Zia, A. I., Rahman, M. S. A., Mukhopadhyay, S. C., Yu, P. L., Al-Bahadly, I. H., and Gooneratne, C. P. et al. 2013. "Technique for Rapid Detection of Phthalates in Water and Beverages." J. Food Eng. 116: 515-523.

[8] Fasano, E., Blay, F. B., Cirillo, T., Montuori, P., and Lacorte, S. 2012. "Migration of Phthalates, Alkylphenols, Bisphenol A and Di(2-ethylhexyl) adipate from Food Packaging." Food Control 27: 132-138.

[9] Mutsuga, M., Tojima, T., Kawamura, Y., and Tanamoto, K. 2005. "Survey of Formaldehyde, Acetaldehyde And Oligomers in Polyethylene Terephthalate Food-Packaging Materials." Food Addit. Contam. 22: 783-789.

[10] Higuchi, A., Yoon, B. O., Kaneko, T., Hara, M.,
Maekawa, M., and Nohmi, T. 2004. "Separation of Endocrine Disruptors from Aqueous Solutions by Pervaporation: Dioctylphthalate and Butylated Hydroxytoluene in Mineral Water." J. Appl. Polym. Sci. 94: 1737-1742.

[11] Hirayama, K., Tanaka, H., Kawana, K., Tani, T., and Nakazawa, H. 2001. "Analysis of Plasticizers in Cap-Sealing Resins for Bottled Foods." Food Addit. Contam. 18: 357-362.

[12] Leivadara, S. V., Nikolaou, A. D., and Lekkas, T. D. 2008. "Determination of Organic Compounds in Bottled Waters." Food Chem. 108: 277-286.

[13] Montuori, P., Jover, E., Morgantini, M., Bayona, J. M., and Triassi, M. 2008. "Assessing Human Exposure to Phthalic Acid and Phthalate Esters from Mineral Water Stored in Polyethylene Terephthalate and Glass Bottles." Food Addit. Contam. 25: 511-518.

[14] Fankhauser-Noti, A., and Grob, K. 2007. "Blank Problems in Trace Analysis of Diethylhexyl and Dibutyl Phthalate: İnvestigation of the Sources, Tips and Tricks." Anal. Chim. Acta. 582: 353-360.

[15] Casajuana, N., and Lacorte, S. 2003. "Presence and Release of Phthalic Esters and Other Endocrine Disrupting Compounds in Drinking Water." Chromatographia 57: 649-655.

[16] Zweifel, H. 2001. Handbook of Plastic Additives, 5th Ed. Munich (Allemagne): Hanser Publications.

[17] Sheftel, V. O. 2000. Indirect Food Additives and Polymers: Migration and Toxicology. USA: CRC Press.

[18] Tombesi, N. B., Freije, R. H., and Augusto, F. 2004. "Factorial Experimental Design Optimization of Solid Phase Microextraction (SPME) Conditions for Analysis of Butylated Hydroxytoluene (BHT) in Bottled Water." Journal of the Brazilian Chemical Society 15: 658-663.

[19] Higuchi, A., Yoon, B. O., Kaneko, T., Hara, M., Maekawa, M., and Nohmi, T. 2004. "Separation of Endocrine Disruptors from Aqueous Solutions by Pervaporation: Dioctylphthalate and Butylated Hydroxytoluene in Mineral Water." J. Appl. Polym. Sci. 94: 1737-1742.

[20] Chimassorb 81. 2011. MSDS No. 30472796. New Jersey: Basf Corporation.

[21] Oleamide. 2003. MSDS No. 301-02-0. USA: Crompton Corporation.

[22] Antioxidant 2246. 1993. MSDS No. BXRNK. USA: Cytec Industries Inc..

[23] Babich, H. 1982. "Butylated Hydroxytoluene (BHT): A Review." Environ. Res. 29: 1-29. 